\title{
Caracterização dos períodos seco e chuvoso da cidade de Fortaleza (CE)
}

\author{
Characterization of dry and wet periods of Fortaleza city (CE)
}

\author{
Ícaro Jael Mendonça Moura ${ }^{1}$, Daiane Fabrício dos Santos ${ }^{1}$, Francisco Geraldo de Melo \\ Pinheiro $^{2}$ e Carlos Jacinto de Oliveira ${ }^{2}$
}

${ }^{1}$ Bolsista de IC, Curso de Graduação em Física, Universidade Estadual do Ceará

${ }^{2}$ Mestrado em Ciências Físicas Aplicadas do Centro de Ciências e Tecnologia da Universidade Estadual do Ceará

\begin{abstract}
Resumo
As condições meteorológicas e climáticas, regionais e globais refletem na biosfera e em muitas atividades e rotinas da humanidade, tais como: agricultura, consumo de energia, risco de desastres naturais, etc. As chuvas, de modo particular, representam um influente fator no planejamento destas várias atividades e rotinas. Portanto, a previsão correta dos seus valores mais prováveis, das áreas de ocorrência e dos períodos em que se distribuem são informações imprescindíveis para o planejamento hídrico de uma região. O objetivo do estudo aqui apresentado é a caracterização de dois períodos meteorológicos bem definidos na Cidade de Fortaleza, Capital do Estado do Ceará, Brasil - o período seco e o chuvoso. É propalado que na Cidade de Fortaleza o período chuvoso ocorre no primeiro semestre do ano (janeiro a junho) e se concentra na quadra mensal de fevereiro a maio, enquanto que o período seco ocorre no segundo semestre do ano (julho a dezembro) e se concentra na quadra mensal de agosto a novembro. Neste estudo, foram utilizados dados de acumulados mensais de precipitação pluviométrica, disponibilizados pelo Sistema de Geração e Disponibilização de Informações Climatológicas (SGDIC) do Instituto de Controle do Espaço Aéreo (ICEA), e coletados por um período de 62 anos (1951 a 2012) numa estação meteorológica localizada no Aeroporto Internacional Pinto Martins, na Cidade de Fortaleza (CE). A partir destes dados, e utilizando um método que define meses secos e chuvosos, em comparação com a mediana da série temporal, a informação propalada foi evidenciada.
\end{abstract}

Palavras-chave: Clima, pluviosidade. Caracterização pluviométrica. Período seco. Período chuvoso.

\begin{abstract}
Meteorological and climatic, regional and global, conditions are reflected on the biosphere and on many human activities and routines such as agriculture, energy consumption, risk of natural disasters, etc. Particularly, rainfall represents an influential factor in the planning of these activities and routines. Therefore, the correct prediction of its most probable values, the occurrence areas and periods in which they are distributed are essential information for a region's water resource management. This paper aims to characterize two well-defined periods for Fortaleza City, the Ceará State's Capital, Brazil - the dry and wet periods. It is said that, in Fortaleza City, the pluviometric wet season occurs in the first half of the year (from January to June) and is more intense on the four-month period from February to May while the pluviometric dry season occurs during the second half of the year (from July to December) and is more intense on the four-month period from August to November. Monthly accumulated precipitation data was used for this study. This data was collected over a period of 62 years (1951-2012) on a weather station located in Pinto Martins International Airport in Fortaleza City (CE) and provided by the SGDIC (Climatic Information Generation System) from the ICEA (Airspace Control Institute). The said information was evidenced using a method that defines dry and rainy months by comparison with the median of the time series.
\end{abstract}

Keywords: Climate, Rainfall. Pluviometric characterization. Dry period. Wet period 


\section{Introdução}

As condições meteorológicas e climáticas, regionais e globais refletem na biosfera e em muitas atividades e rotinas da humanidade, tais como agricultura, consumo de energia, risco de desastres naturais, etc. A grande diversidade entre as regiões pode, em grande parte, ser explicada pelos vários fatores que atuam em seus climas, combinadamente, fatores locais, regionais e de escala global (SANTOS, 2000). Em condições ótimas, o clima e as suas variações exercem influências positivas sobre as sociedades através da precipitação pluvial, radiação solar, vento, etc. Entretanto, em condições adversas, manifestadas pela ocorrência de enchentes, tempestades, secas, granizo, entre outras, influencia de modo negativo. A ocorrência das anomalias climáticas mostra que a humanidade e suas atividades econômicas, principalmente as ligadas à agricultura, são vulneráveis a tais fenômenos (SOUSA, 2006).

As chuvas, de modo particular, representam um influente fator no planejamento de várias atividades humanas e, ocorrendo em quantidade adequada e num período ótimo, beneficiam enormemente o cultivo, crescimento, amadurecimento das plantas e o rendimento das atividades associadas à agricultura, à pesca, à pecuária e, também, ao abastecimento de água para consumo. Por outro lado, sua ausência, enfraquecimento, ou ainda desaparecimento temporário, os chamados veraneios, em períodos em que se espera o contrário estão sempre associados a grandes prejuízos econômicos (BERLATO et al., 1992; BERLATO; FONTANA, 1999; CUNHA et al., 1999). A previsão correta de seus valores mais prováveis, bem como das áreas de ocorrência e regularidade que as mesmas se distribuem, são informações impres- cindíveis para o planejamento hídrico de uma região (SALGUEIRO, 2005).

Portanto, torna-se importante, se não necessário e fundamental, o estudo do comportamento das chuvas das diversas áreas de cada região do globo terrestre, de forma que ações de caráter preventivo possam ser tomadas em tempo adequado, diminuindo tais prejuízos.

É propalado que, na Cidade de Fortaleza (CE), existem dois períodos pluviométricos bem definidos - o período chuvoso, que ocorre durante o primeiro semestre do ano e é mais intenso na quadra mensal de fevereiro a maio, e o período seco, que ocorre durante o segundo semestre do ano e é mais intenso na quadra mensal de agosto a novembro. Seriam esses os períodos em que a precipitação ocorre mais frequentemente, ou menos frequentemente, nessa cidade?

Os objetivos da pesquisa apresentada neste artigo foram investigar a veracidade da informação propalada acerca dos períodos pluviométricos e caracterizar, utilizando dados de precipitação pluviométrica históricos, os períodos pluviométricos da Cidade de Fortaleza.

\section{Materiais e Métodos}

\subsection{Região de Estudo}

A Cidade de Fortaleza, Capital do Estado do Ceará, Brasil, localizada nas coordenadas $3^{\circ} 47^{\prime} \mathrm{S}$ e $38^{\circ} 30^{\prime} \mathrm{O}$, com área de $313,8 \mathrm{~km}^{2}$ e população de dois milhões e quinhentos mil habitantes, possui a maior densidade demográfica do país, 7815,7 hab/ $\mathrm{km}^{2}$ (IBGE, 2010); ver Figura 1. A Cidade possui uma área muito urbanizada (Figura 2) e é litorânea, com 24 km de praias do leste

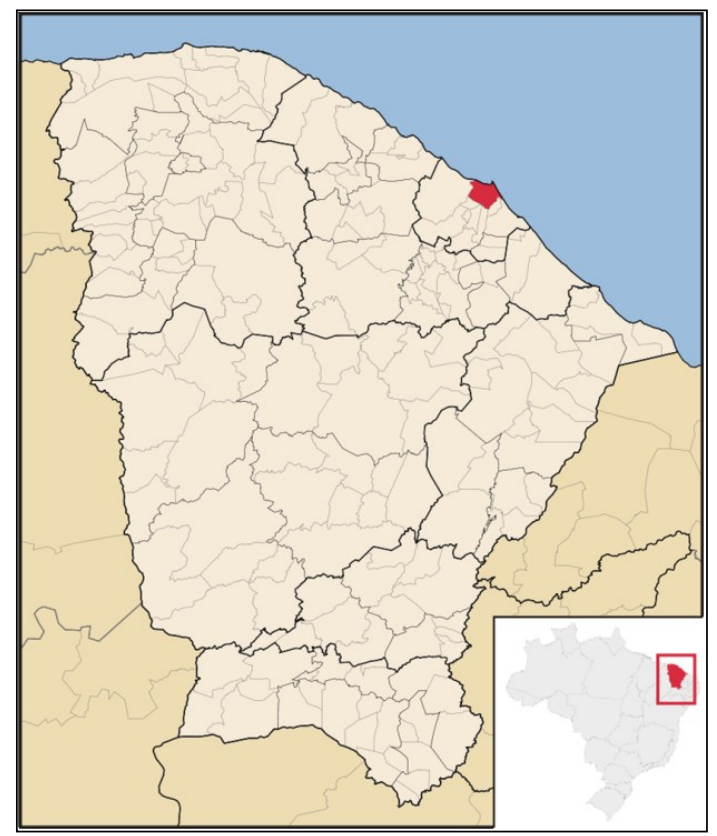

Figura 1 - Mapa Cartográfico do Ceará, Brasil, com destaque (em vermelho) para o Estado do Ceará e para a Cidade de Fortaleza; Autor: Darlan P. de Campos 


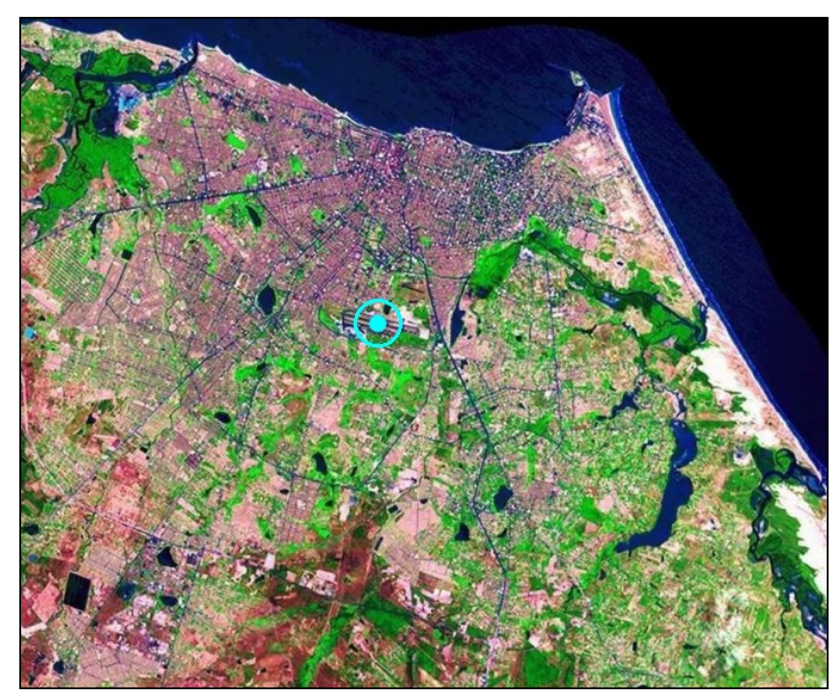

Figura 2 - Imagem da Cidade de Fortaleza, com destaque para construções (em cor roxa), para vegetação (em cor verde), para vias (em cor azul) e para a posição da estação meteorológica utilizada em círculo de cor ciano, com circunferência concêntrica

Fonte: Adaptado de Miranda e Coutinho (2004)

do Continente da América do Sul, banhada pelo Oceano Atlântico.

\subsection{Coleta de Dados}

Foram utilizados dados de precipitação pluviométrica acumulada mensal, disponibilizados pelo Sistema de Geração e Disponibilização de Informações Climatológicas do Instituto de Controle do Espaço Aéreo (ICEA). Esses dados foram coletados por um período de 62 anos, de 1951 até 2012, em estação meteorológica automática fixa localizada no Aeroporto Internacional Pinto Martins, latitude $-3,36^{\circ}$ e longitude $-38,32^{\circ}$ em altitude de $25 \mathrm{~m}$, na Cidade de Fortaleza.

Sendo esse um período consideravelmente longo de coleta, é certo que a instrumentação usada nas medições sofreu mudanças ao longo desse período e, portanto, ocorreram falhas na série de dados. Entretanto, a grande quantidade de dados coletados e o grande período de tempo no qual foram realizadas as medições garantem, estatisticamente, a consistência dos resultados. As falhas na série de dados eram pouco numerosas (22 meses sem dados em um total de 744 meses na série, representando menos de $3 \%$ de falha). Neste trabalho, tais problemas foram desconsiderados.

\subsection{Métodos}

Para a definição de meses secos e chuvosos, utilizouse um dos métodos propostos por Gregory (1979) em escala de tempo mensal. De acordo com esse método, definem-se mês seco e mês chuvoso como sendo aqueles cujo valor da pluviosidade é menor e maior que a mediana da série, respectivamente.

Deste modo, construiu-se uma tabela de contingência da classificação dos meses do ano em secos ou chuvosos. A significância estatística da classificação dos meses foi calculada utilizando o Teste Exato de Fisher (TEF).

O TEF é um método utilizado para testar a significância da associação entre tipos de classificação. Neste trabalho, os tipos de classificação considerados foram mês seco ou mês chuvoso para todos os meses do ano (janeiro, fevereiro, etc.).

No TEF calcula-se a probabilidade condicional da tabela de contingência (considerada uma matriz) por

$$
P=\frac{\left(\prod_{i=1}^{i=m} R_{i} !\right)\left(\prod_{j=1}^{j=n} C_{j} !\right)}{N !\left(\prod_{i=1, j=1}^{i=m, j=n} a_{i, j} !\right)}
$$

onde $R_{i}$ e $C_{j}$ representam, respectivamente, o somatório dos valores da $i$-ésima linha e $j$-ésima coluna da matriz de $m$ linhas e $n$ colunas; e $N$ é o somatório dos elementos $a_{i, j}$ da matriz. Finalmente, o p-valor do teste é calculado como sendo a soma de todas as probabilidades condicionais $P^{\prime}$, de todas as matrizes que podem ser criadas a partir da tabela de contingência ao se variar os $a_{i, j}$ mantendo-se constante, que obedeçam $P \geq P^{\prime}$ (FISHER, 1925).

\section{Resultados e Discussão}

A Tabela 1 apresenta a tabela de contingência dos meses chuvosos e dos meses secos, conforme a classificação proposta por Gregory. Observa-se uma grande frequência de meses chuvosos no período de janeiro a junho, com ocorrência mínima de 39 em janeiro e máxima de 58 em março, e uma grande frequência de meses 
Tabela 1 - Tabela de contingência da classificação dos meses do ano quanto a serem secos ou chuvosos

\begin{tabular}{|l|c|c|c|c|c|c|c|c|c|c|c|c|}
\hline & Jan & Fev & Mar & Abr & Mai & Jun & Jul & Ago & Set & Out & Nov & Dez \\
\hline Chuvosos & 39 & 51 & 58 & 55 & 56 & 45 & 27 & 5 & 6 & 4 & 1 & 14 \\
\hline Secos & 20 & 9 & 3 & 5 & 5 & 15 & 31 & 56 & 54 & 56 & 60 & 47 \\
\hline
\end{tabular}

Tabela 2 - Valores das PAMM e dos respectivos Desvios Padrões dos meses do ano no período de estudo

\begin{tabular}{|l|c|c|c|c|c|c|c|c|c|c|c|c|}
\hline & Jan & Fev & Mar & Abr & Mai & Jun & Jul & Ago & Set & Out & Nov & Dez \\
\hline PAAM & 123,36 & 192,56 & 362,59 & 384,67 & 242,41 & 157,26 & 72,65 & 33,16 & 27,39 & 32,49 & 12,30 & 37,79 \\
\hline $\begin{array}{l}\text { Desvio } \\
\text { Padrão }\end{array}$ & 109,34 & 207,84 & 304,08 & 352,56 & 328,28 & 160,27 & 74,35 & 81,12 & 50,54 & 114,40 & 17,34 & 73,92 \\
\hline
\end{tabular}

secos no período de julho a dezembro, com ocorrência mínima de 31 em julho e máxima de 60 em novembro.

Verifica-se, assim, que a informação propalada é evidenciada pela climatologia da pluviosidade na cidade, utilizando o método proposto por Gregory, uma vez que os meses considerados chuvosos se apresentam mais frequentemente $(84 \%)$ nos meses do primeiro semestre do ano (de janeiro a junho) e os meses considerados secos se apresentam mais frequentemente $(84 \%)$ nos meses do segundo semestre do ano (de julho a dezembro). A significância estatística deste resultado, calculada pelo TEF, obedece $p<0,05$.

A Tabela 2 apresenta os valores calculados da Pluviosidade Acumulada Mensal Média (PAMM) e dos respectivos desvios padrões para cada mês no período de estudo. A Figura 3 mostra o gráfico desses valores.

Na Figura 3, e de acordo com as Tabelas 1 e 2, observa-se que no período chuvoso, que segundo a classificação de Gregory, ocorre em todo o primeiro semestre, a PAMM na região de estudo é bem elevada, variando de um mínimo de 123,36 mm, em janeiro, a um máximo de $384,67 \mathrm{~mm}$, em abril. O período seco, que segundo a mesma classificação ocorre em todo o segundo semestre, apresenta PAMM baixas, variando de um mínimo de $12,30 \mathrm{~mm}$, em novembro, a um máximo de $72,65 \mathrm{~mm}$, em julho.

Adicionalmente, são evidenciadas as informações propaladas acerca da quadra mensal chuvosa e da quadra mensal seca. A quadra mensal de fevereiro a maio, propalada de "quadra chuvosa", apresenta, em média, o maior acumulado de pluviosidade, $1182,22 \mathrm{~mm}$, dentre qualquer outra quadra mensal; semelhantemente, a quadra mensal de agosto a novembro, propalada de "quadra seca", apresenta, em média, o menor acumulado de pluviosidade, $105,35 \mathrm{~mm}$.

Ainda no gráfico da Figura 3, pode-se observar que a PAMM aumenta a partir de janeiro, $123,36 \mathrm{~mm}$, até abril, $384,67 \mathrm{~mm}$, que, em média, é o mês mais chuvoso do ano e, então, diminui até setembro, 27,39 mm. Quebrando essa sequência de diminuição, em outubro há um aumento da PAMM, passando dos $27,39 \mathrm{~mm}$ de setembro a 32,49 $\mathrm{mm}$, pluviosidade esta popularmente conhecida como

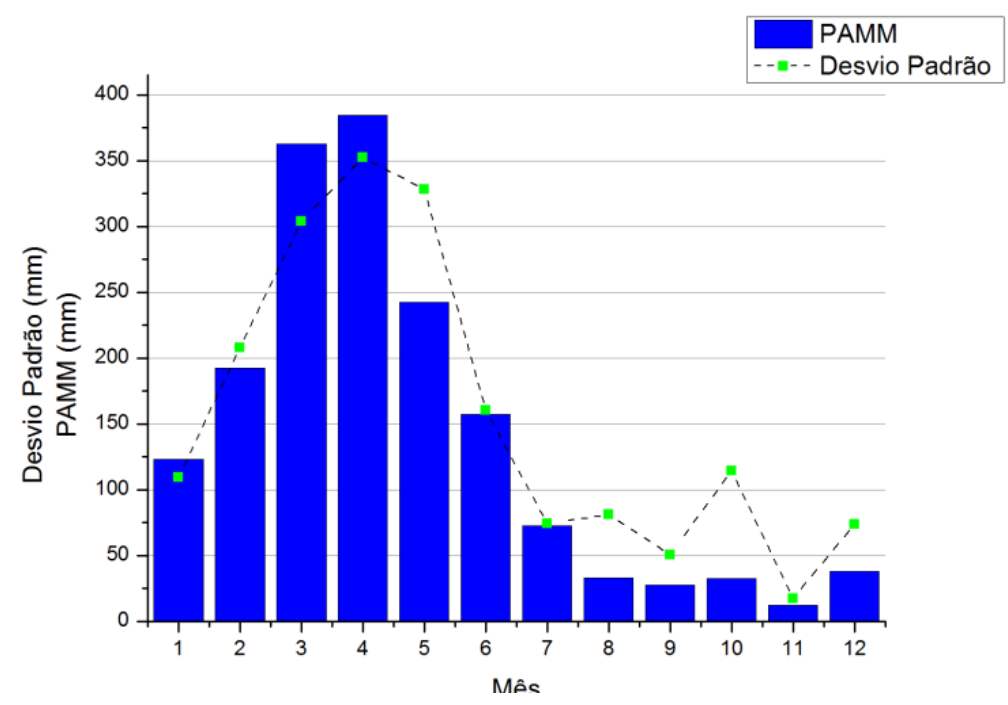

Figura 3 - Gráfico da PAMM, em colunas de cor azul, e do seu Desvio Padrão, em pontos de cor verde interligados por linha tracejada, no período de estudo 
"chuva do caju", e diminuindo novamente em novembro, 12,30 mm, que, em média, é o mês mais seco do ano. Em dezembro, a PAMM volta a aumentar.

Observa-se, também, que a variabilidade da pluviosidade, apresentada no gráfico na forma de desvio padrão, é bem elevada. Nos meses em que a PAMM é elevada, a variabilidade é também elevada. Isso pode ser devido a uma distribuição altamente irregular das chuvas no tempo e no espaço.

Combinando os resultados da Tabela 1 e da Figura 3, podem ser identificados meses com valores de PAMM intermediários entre os da quadra chuvosa e os da quadra seca. Esses meses são aqui classificados como "períodos de transição". São eles: junho e julho, chamados de "pós -estação chuvosa" e dezembro e janeiro, chamados de "pré-estação chuvosa".

\section{Conclusões}

O teste de classificação de meses em secos e chuvosos, proposto por Gregory (1979), evidencia a informação propalada acerca da pluviosidade na Cidade de Fortaleza: o período chuvoso acontece no primeiro semestre do ano e o período seco no segundo semestre.

Na Cidade de Fortaleza, durante o período chuvoso, a PAMM é bastante elevada, variando de um mínimo de 123,36 mm em janeiro a um máximo de 384,67 mm em abril. No período seco, entretanto, a PAMM é reduzida, variando de um mínimo de $12,30 \mathrm{~mm}$ em novembro a um máximo de 72,65 mm em julho. Assim, o mês de abril e o mês de novembro são, respectivamente, em média, o mais chuvoso e o mais seco do ano.

São evidenciadas as informações propaladas acerca da "quadra chuvosa" (de fevereiro a maio) e da "quadra seca" (de agosto a novembro) sendo estas, em média, as quadras mensais com maior, $1182,22 \mathrm{~mm}$, e menor, 105,35 mm, acumulado de pluviosidade.

Em outubro, observa-se uma quebra na diminuição da PAMM no período seco com um aumento do seu valor. Isto caracteriza a chamada "chuva do caju" da cidade de Fortaleza.

São identificados dois períodos de transição: a "pós -estação chuvosa" composta pelos meses de junho e julho, e a "pré-estação chuvosa", composta pelos meses de dezembro e janeiro.

\section{Agradecimentos}

Os autores agradecem ao CNPq pelo auxílio financeiro em forma de Bolsa de Iniciação Científica e ao ICEA pela disponibilização dos dados.

\section{Referências}

BERLATO, M. A.; FONTANA, D. C.; GONÇALVES, H. M. Relação entre o rendimento de grãos de soja e variáveis meteorológicas. Pesquisa Agropecuária Brasileira, 1992.

BERLATO, M. A.; Fontana, D. C. Variabilidade interanual da precipitação e variabilidade dos rendimentos de soja no Estado do Rio Grande do Sul. Revista Brasileira de Agrometeorologia, 1999.

CUNHA, G. R.; DALMAGO, G. A.; ESTEFANEL, V. Enso influences on wheat crop in Brazil. Revista Brasileira de Agrometorologia, 1999.

FISHER, R. A. Statistical Methods for Research Workers. Edinburgh: Oliver and Boyd, 1925.

GREGORY, S. The definition of wet and dry periods for discrete regional units, Weather, 34, 1979.

\section{IBGE-INSTITUTO BRASILEIRO DE GEOGRAFIA E ESTATÍSTICA. Censo 2010, 2010.}

MIRANDA, E. E. de; COUTINHO, A. C. Brasil Visto do Espaço. Campinas: Embrapa Monitoramento por Satélite, 2004. Disponível em: http://www.cdbrasil.cnpm. embrapa.br. Acesso em: 3 jul. 2008.

SALGUEIRO, J. H. P. B. Avaliação de rede pluviométrica e análise de variabilidade espacial da precipitação: estudo de caso na Bacia do Rio Ipojuca em Pernambuco. Dissertação (mestrado) - Universidade Federal de Pernambuco. CTG. Engenharia Civil. Recife-PE, 2005.

SANTOS, S. M dos; LEAL, L. S. M.; SILVA, M. M. da. Caracterização de Estação Seca e chuvosa para a Cidade de Viçosa-MG.. In: CONGRESSO BRASILEIRO DE METEOROLOGIA, 11, Rio de Janeiro, 2000. Anais. Rio de Janeiro, Sociedade Brasileira de Meteorologia, 2000.

SOUSA, P. Estudo da variabilidade da precipitação no estado do Paraná associado à anomalia da TSM no Oceano Pacífico. Dissertação de mestrado. Universidade Estadual de Maringá - “UEM",2006. Disponível em: http://www. pge.uem.br/novo/teses/pdf/psouza.pdf 